PROCEEDINGS OF THE

AMERICAN MATHEMATICAL SOCIETY

Volume 128, Number 2, Pages 401-405

$\mathrm{S} 0002-9939(99) 05424-6$

Article electronically published on September 9, 1999

\title{
THE FIRST OCCURRENCE FOR THE IRREDUCIBLE MODULES OF GENERAL LINEAR GROUPS IN THE POLYNOMIAL ALGEBRA
}

\author{
PHAM ANH MINH AND TON THAT TRI
}

(Communicated by Ronald M. Solomon)

\begin{abstract}
Let $p$ be a prime number and let $G L_{n}$ be the group of all invertible matrices over the prime field $\mathbb{F}_{p}$. It is known that every irreducible $G L_{n^{-}}$ module can occur as a submodule of $P$, the polynomial algebra with $n$ variables over $\mathbb{F}_{p}$. Given an irreducible $G L_{n}$-module $\rho$, the purpose of this paper is to find out the first value of the degree $d$ of which $\rho$ occurs as a submodule of $P_{d}$, the subset of $P$ consisting of homogeneous polynomials of degree $d$. This generalizes Schwartz-Tri's result to the case of any prime $p$.
\end{abstract}

\section{INTRODUCTION}

Let $p$ be a prime number and let $G L_{n}=G L\left(n, \mathbb{F}_{p}\right)$ be the group of all invertible $(n \times n)$-matrices over the prime field $\mathbb{F}_{p}$. Denote by $P=\mathbb{F}_{p}\left[x_{1}, \ldots, x_{n}\right]$ the polynomial algebra with variables $x_{1}, \ldots, x_{n}$ over $\mathbb{F}_{p}$. There is then an action of $G L_{n}$ on $P$ given by

$$
\begin{aligned}
{ }^{\sigma} x_{i} & =\sum_{j=1}^{n} a_{j i} x_{j}, \quad 1 \leq i \leq n, \\
{ }^{\sigma} f\left(x_{1}, \ldots, x_{n}\right) & =f\left({ }^{\sigma} x_{1}, \ldots,{ }^{\sigma} x_{n}\right)
\end{aligned}
$$

with $\sigma=\left(a_{i j}\right)_{1 \leq i, j \leq n} \in G L_{n}, f\left(x_{1}, \ldots, x_{n}\right) \in P$.

For an irreducible $G L_{n}$-module $\rho$, it is known (see e.g. [1]) that $\rho$ is isomorphic to a submodule of $P$. One may ask about the first value of the degree $d$ for which $\rho$ occurs as a submodule of $P_{d}$, the subset of $P$ consisting of homogeneous polynomials of degree $d$. This first occurrence problem also arises from topology, as it is related to the description of the cohomology of certain spaces as modules over the Steenrod algebra (see e.g. [7]). For the prime 2, the problem has been solved independently by Schwartz [4] and Tri [6]. The purpose of this paper is to solve this problem by generalizing the argument used in [6] to the case of any prime $p$.

Let us recall (see e.g. [2, Chapter 8]) that the irreducible $G L_{n}$-modules are indexed, up to isomorphism, by column regular partitions, i.e., by sequences of non-negative integers

$$
\alpha_{1}, \ldots, \alpha_{n}
$$

Received by the editors April 10, 1998.

1991 Mathematics Subject Classification. Primary 20C20.

(C)1999 American Mathematical Society 
satisfying $0 \leq \alpha_{i}-\alpha_{i+1} \leq p-1$ for $1 \leq i \leq n-1$ and $0 \leq \alpha_{n} \leq p-1$. Namely, the irreducible $G L_{n}$-modules are indexed by column regular partitions $\alpha_{1}, \ldots, \alpha_{n}$ satisfying $\alpha_{n} \leq p-2$. Recently, in [5], they were also obtained by means of modular invariants, as follows. For $1 \leq i \leq n$, let

$$
V_{i}=\prod_{\lambda_{j} \in \mathbb{F}_{p}}\left(\lambda_{1} x_{1}+\cdots+\lambda_{i-1} x_{i-1}+x_{i}\right)
$$

be the Mui invariant and let

$$
L_{i}=V_{1} \cdots V_{i}
$$

be the Dickson invariant. It is clear that

$$
L_{n}=\left|\begin{array}{cccc}
x_{1} & x_{2} & \ldots & x_{n} \\
x_{1}^{p} & x_{2}^{p} & \ldots & x_{n}^{p} \\
\dot{\cdot} & \dot{\cdot} & \ldots & \cdot \\
x_{1}^{p^{n-1}} & x_{2}^{p^{n-1}} & \ldots & x_{n}^{p^{n-1}}
\end{array}\right|
$$

and ${ }^{\sigma} L_{n}=\operatorname{det} \sigma \cdot L_{n}$ for $\sigma \in G L_{n}$. Let $\beta=\left(\beta_{1}, \ldots, \beta_{n}\right)$ with $0 \leq \beta_{i} \leq p-1$ and set $L^{\beta}=\prod_{i=1}^{n} L_{i}^{\beta_{i}} \in P$. Denote by $H_{\beta}=H_{\beta}\left(G L_{n}\right)$ the $G L_{n}$-module generated by $L^{\beta}$ (so $H_{\beta}$ is nothing but the $\mathbb{F}_{p^{-}}$-vector space generated by the set $\left\{{ }^{\sigma} L^{\beta} \mid \sigma \in G L_{n}\right\}$ ). We have

Theorem A $\left(\left[5\right.\right.$, Corollary 1.2]). $\left\{H_{\beta} \mid \beta=\left(\beta_{1}, \ldots, \beta_{n}\right), 0 \leq \beta_{i} \leq p-1, \beta_{n} \neq 0\right\}$ is a complete set of $(p-1) p^{n-1}$ distinct irreducible modules for the algebra $\mathbb{F}_{p}\left[G L_{n}\right]$.

By noting that $H_{\left(\beta_{1}, \ldots, \beta_{n-1}, p-1\right)} \cong H_{\left(\beta_{1}, \ldots, \beta_{n-1}, 0\right)}$, we can restate the theorem as follows.

Theorem B (compare $\left[1\right.$, Proposition 1.3]). $\left\{H_{\beta} \mid \beta=\left(\beta_{1}, \ldots, \beta_{n}\right), 0 \leq \beta_{i} \leq p-\right.$ $\left.1, \beta_{n} \neq p-1\right\}$ is a complete set of $(p-1) p^{n-1}$ distinct irreducible modules for the algebra $\mathbb{F}_{p}\left[G L_{n}\right]$.

We should note that, in terms of column regular partitions, for $0 \leq \beta_{i} \leq p-$ $1, \beta_{n} \neq p-1, H_{\beta}$ corresponds to the sequence $\lambda_{1}, \ldots, \lambda_{n}$ with $\lambda_{i}=\beta_{i}+\cdots+\beta_{n}, 1 \leq$ $i \leq n$.

Generalizing Schwartz-Tri's result ([4], [6]) to the case of any prime, we prove

Theorem C. With $\beta$ given in Theorem $B$, the first occurrence of $H_{\beta}$ as a submodule of $P_{d}$ is for $d=\operatorname{deg} L^{\beta}$.

\section{Preliminaries}

Let $T_{n}$ be the group consisting of upper triangular matrices with 1 on the diagonal. It follows from [3] that

$$
P^{T_{n}}=\mathbb{F}_{p}\left[V_{1}, \ldots, V_{n}\right] .
$$

We shall use the following notation. Given $g=g\left(x_{1}, \ldots, x_{n}\right) \in P, \lambda \in \mathbb{F}_{p}$ and $1 \leq i, k \leq n$, then ${ }^{\sigma_{i, k, \lambda}} g$ (resp. ${ }^{\eta_{i, \lambda}} g,{ }^{\tau_{i, k}} g$ ) denotes the polynomial obtained from $g$ by replacing $x_{i}$ by $x_{i}+\lambda x_{k}$ (resp. by replacing $x_{i}$ by $\lambda x_{i}$, by interchanging $x_{i}$ and $\left.x_{k}\right)$. Consider a homogeneous element $f=f\left(x_{1}, \ldots, x_{n}\right)$ of $P^{T_{n}}$. It is clear that $V_{i}$ is a factor of $f$ if and only if $x_{i}$ is also. We have

Lemma 1. If $\eta_{i, \lambda} f=\lambda^{\ell} f$ with $1 \leq \ell<p-1$ and $\lambda \neq 0,1$, then $f$ contains $x_{i}$ as a factor. 
Proof. Write $f=\sum_{r=0}^{m} x_{i}^{r} f_{r}\left(x_{1}, \ldots, x_{n}\right)$ with $f_{r}$ free of $x_{i}$. It follows that $\sum_{r=0}^{m} x_{i}^{r}\left(\lambda^{\ell} f_{r}-\lambda^{r} f_{r}\right)=0$. So $\lambda^{\ell} f_{0}-f_{0}=0$, which implies that $f_{0}=0$. Therefore $f$ contains $x_{i}$ as a factor. The lemma follows.

The case $p=2$ in the following lemma was treated in [6]. However, our proof given here, for any prime $p$, is much simpler.

Lemma 2. If $i<k$, then $f$ contains $x_{i}$ as a factor provided that one of the following conditions is satisfied:

(i) $\sum_{r=0}^{p-1} \sigma_{i, k, r} f=-\tau_{i, k} f$ and $\eta_{i, \mu} f=f$ for every $\mu \neq 0$;

(ii) $f$ contains $x_{k}$ as a factor and $\sum_{r=0}^{p-1} \sigma_{i, k, r} f=\alpha \cdot{ }^{\tau_{i, k}} f$ with $\alpha \in\{0,1,-1\}$.

Proof. For $1 \leq r \leq p-1$, since $f$ is $T_{n}$-invariant, we have

$$
\begin{aligned}
f( & \left.x_{1}, \ldots, x_{i-1}, r x_{k}, x_{i+1}, \ldots, x_{k}, \ldots, x_{n}\right) \\
& =f\left(x_{1}, \ldots, x_{i-1}, r x_{k}, x_{i+1}, \ldots, x_{k}-x_{k}, \ldots, x_{n}\right) \\
& =f\left(x_{1}, \ldots, x_{i-1}, r x_{k}, x_{i+1}, \ldots, 0, \ldots, x_{n}\right) \\
& = \begin{cases}f\left(x_{1}, \ldots, x_{i-1}, x_{k}, x_{i+1}, \ldots, 0, \ldots, x_{n}\right), & \text { if } \eta_{i, r} f=f, \\
0, & \text { if } f \text { contains } x_{k} \text { as a factor. }\end{cases}
\end{aligned}
$$

Consider $\sum_{r=0}^{p-1} \sigma_{i, k, r} f$ and set $x_{i}=0$. (i) and the above equality imply that

$$
\begin{aligned}
\sum_{r=0}^{p-1} \sigma_{i, k, r} f= & f\left(x_{1}, \ldots, x_{i-1}, 0, x_{i+1}, \ldots, x_{k}, \ldots, x_{n}\right) \\
& -f\left(x_{1}, \ldots, x_{i-1}, x_{k}, x_{i+1}, \ldots, 0, \ldots, x_{n}\right) \\
= & -f\left(x_{1}, \ldots, x_{i-1}, x_{k}, x_{i+1}, \ldots, 0, \ldots, x_{n}\right)
\end{aligned}
$$

so $f\left(x_{1}, \ldots, x_{i-1}, 0, x_{i+1}, \ldots, x_{k}, \ldots, x_{n}\right)=0$; on the other hand, (ii) implies that

$$
\begin{aligned}
\sum_{r=0}^{p-1} \sigma_{i, k, r} f & =f\left(x_{1}, \ldots, x_{i-1}, 0, x_{i+1}, \ldots, x_{k}, \ldots, x_{n}\right) \\
& =\alpha f\left(x_{1}, \ldots, x_{i-1}, x_{k}, x_{i+1}, \ldots, 0, \ldots, x_{n}\right) \\
& =0, \quad \text { since } f \text { contains } x_{k} \text { as a factor. }
\end{aligned}
$$

In any case, $f$ contains $x_{i}$ as a factor. The lemma follows.

\section{Proof of Theorem C}

We proceed by induction on $n$. The proof is trivial for $n=1$. Assume that the theorem holds for $n-1$.

Let $W$ be an irreducible $G L_{n}$-module of homogeneous polynomials. There exist then $\beta=\left(\beta_{1}, \ldots, \beta_{n}\right)$ with $0 \leq \beta_{i} \leq p-1, \beta_{n} \neq p-1$ and a $G L_{n}$-isomorphism $\zeta: W \rightarrow H_{\beta}\left(G L_{n}\right)$. Set $f=f\left(x_{1}, \ldots, x_{n}\right)=\zeta^{-1}\left(L^{\beta}\right)$. We need to prove that $\operatorname{deg} f \geq \operatorname{deg} L^{\beta}$. It is clear that $f$ is invariant under the action of $T_{n}$ and $W$ is the $G L_{n}$-module generated by $f$. For convenience, write $W=W_{f}\left(G L_{n}\right)$.

Denote by $\left(\beta_{n_{1}}, \ldots, \beta_{n_{k}}\right)$ the subsequence of $\beta$ consisting of all non-zero elements, so $L^{\beta}=L_{n_{1}}^{\beta_{1}} \cdots L_{n_{k}}^{\beta_{n_{k}}}$. First, we prove that $f$ contains $L_{n_{k}}$ as a factor (note that $\beta_{n_{k}}<p-1$ if $n_{k}=n$ ). Let $m$ be an integer satisfying $n_{k-1}<m \leq n_{k}$. For every $\mu \neq 0$, as ${ }^{\eta_{m, \mu}} L^{\beta}=\mu^{\beta_{n_{k}}} L^{\beta}$, it follows that ${ }^{\eta_{m, \mu}} f=\mu^{\beta_{n_{k}}} f$. If $\beta_{n_{k}}<p-1$, Lemma 1 
implies that $f$ contains $x_{m}$, therefore $V_{m}$, as a factor. If $\beta_{n_{k}}=p-1$ (which implies $\left.n_{k}<n\right)$, then $\eta_{m, \mu} f=f$ and

$$
\begin{aligned}
\sigma_{m, n, r} L_{n_{k}}=L_{n_{k}}\left(x_{1}, \ldots, x_{m-1}, x_{m}+r x_{n}, x_{m+1}, \ldots, x_{n_{k}}\right) \\
=L_{n_{k}}\left(x_{1}, \ldots, x_{m-1}, x_{m}, x_{m+1}, \ldots, x_{n_{k}}\right) \\
\quad+r L_{n_{k}}\left(x_{1}, \ldots, x_{m-1}, x_{n}, x_{m+1}, \ldots, x_{n_{k}}\right)
\end{aligned}
$$

for $r \in \mathbb{F}_{p}$. Since

$$
\begin{aligned}
& \sum_{r=0}^{p-1}\left(L_{n_{k}}\left(x_{1}, \ldots, x_{m-1}, x_{m}, x_{m+1}, \ldots, x_{n_{k}}\right)\right. \\
& \left.\quad+r L_{n_{k}}\left(x_{1}, \ldots, x_{m-1}, x_{n}, x_{m+1}, \ldots, x_{n_{k}}\right)\right)^{\lambda} \\
& \quad= \begin{cases}0, & \text { if } \lambda<p-1, \\
-L_{n_{k}}^{p-1}\left(x_{1}, \ldots, x_{m-1}, x_{n}, x_{m+1}, \ldots, x_{n_{k}}\right), & \text { if } \lambda=p-1,\end{cases}
\end{aligned}
$$

we have $\sum_{r=0}^{p-1}{ }^{\sigma_{m, n, r}} L_{n_{k}}^{p-1}=-L_{n_{k}}^{p-1}\left(x_{1}, \ldots, x_{m-1}, x_{n}, x_{m+1}, \ldots, x_{n_{k}}\right)$ which implies that $\sum_{r=0}^{p-1} \sigma_{m, n, r} L^{\beta}=-{ }^{\tau_{m, n}} L^{\beta}$. Hence $\sum_{r=0}^{p-1} \sigma_{m, n, r} f=-\tau_{m, n} f$. It follows from Lemma 2 that $f$ contains $x_{m}$, hence $V_{m}$, as a factor.

Set $n_{0}=0$. Suppose that $f$ contains $x_{t+1}, \ldots, x_{n_{k}}$ as factors with $n_{\ell-1}<t \leq$ $n_{\ell}<n_{k}$. It follows from $(*)$ that

$$
\sum_{r=0}^{p-1} \sigma_{t, n_{\ell}+1, r} L_{n_{\ell}}^{\beta_{n_{\ell}}}= \begin{cases}0, & \text { if } \beta_{n_{\ell}}<p-1 \\ -{ }^{\tau_{t, n_{\ell}+1}} L_{n_{\ell}}^{p-1}, & \text { if } \beta_{n_{\ell}}=p-1\end{cases}
$$

Therefore

$$
\sum_{r=0}^{p-1} \sigma_{t, n_{\ell}+1, r} L^{\beta}= \begin{cases}0, & \text { if } \beta_{n_{\ell}}<p-1, \\ \alpha^{\tau_{t, n_{\ell}+1}} L^{\beta}, & \text { if } \beta_{n_{\ell}}=p-1,\end{cases}
$$

with $\alpha= \pm 1$. So

$$
\sum_{r=0}^{p-1} \sigma_{t, n_{\ell}+1, r} f= \begin{cases}0, & \text { if } \beta_{n_{\ell}}<p-1, \\ \alpha^{\tau_{t, n_{\ell}+1}} f, & \text { if } \beta_{n_{\ell}}=p-1 .\end{cases}
$$

By Lemma $2, f$ contains $x_{t}$, hence $V_{t}$, as a factor. Therefore $f$ contains $L_{n_{k}}$ as a factor.

Write $f=L_{n_{k}} g$ with $g=g\left(x_{1}, \ldots, x_{n}\right) \in P$. So $g \in P^{T_{n}}$. Consider $G L_{n_{k}}$ as a subgroup of $G L_{n}$ in the usual way. $H_{\beta}\left(G L_{n_{k}}\right)$ and $W=W_{f}\left(G L_{n_{k}}\right)$ are then $G L_{n_{k}}$ modules and $H_{\beta}\left(G L_{n_{k}}\right) \stackrel{\zeta}{\cong} W_{f}\left(G L_{n_{k}}\right) \cong \operatorname{det} \otimes W_{g}\left(G L_{n_{k}}\right)$. Set $\beta^{\prime}=\left(\beta_{1}, \ldots, \beta_{n_{k}}-1\right)$ and $\beta^{\prime \prime}=\left(\beta_{1}, \ldots, \beta_{n_{k-1}}\right)$. As $G L_{n_{k}}$-modules, $H_{\beta}\left(G L_{n_{k}}\right) \cong \operatorname{det} \otimes H_{\beta^{\prime}}\left(G L_{n_{k}}\right)$, so $W_{g}\left(G L_{n_{k}}\right) \cong H_{\beta^{\prime}}\left(G L_{n_{k}}\right)$. If $n_{k}<n$, it follows from the inductive hypothesis that $\operatorname{deg} g \geq \operatorname{deg} L^{\beta^{\prime}}$, so $\operatorname{deg} f \geq \operatorname{deg} L^{\beta}$. Suppose that $n_{k}=n$. The argument used above shows that $g$ contains $L_{n}$ as a factor; hence $f$ contains $L_{n}^{\beta_{n}}$ as a factor.

By writing $f=L_{n}^{\beta_{n}} h$, we have $W_{h}\left(G L_{n}\right) \cong H_{\beta^{\prime \prime}}\left(G L_{n}\right)$. By replacing $f$ by $h$, $\operatorname{arguing}$ as in the case $n_{k}<n$ yields $\operatorname{deg} h \geq \operatorname{deg} L^{\beta^{\prime \prime}}$; hence $\operatorname{deg} f \geq \operatorname{deg} L^{\beta}$. The theorem is proved.

\section{ACKNOWLEDGMENTS}

We thank Grant Walker who introduced us to the study of this first occurrence problem. Part of the result was obtained while the first author visited the University 
of Manchester in Autumn 1997; he thanks Robert Sandling for making the visit possible.

\section{REFERENCES}

1. S. Doty and G. Walker, Truncated symmetric powers and modular representations of $G L_{n}$, Math. Proc. Camb Phil. Soc. 119 (1996), 231-242. MR 96g:20062

2. G.D. James and A. Kerber, The representation theory of the symmetric group, Encyclopedia of Mathematics and its Applications vol. 16 (Addison-Wesley), 1981. MR 83k:20003

3. H. Mui, Modular invariant theory and cohomology algebras of symmetric groups, J. Fac. Sci. Univ. Tokyo Sec. IA 22 (1975), 319-369.

4. L. Schwartz, Unstable modules over the Steenrod algebra and Sullivan's fixed point set conjecture, Chicago Lecture Notes in Mathematics, 1994. MR 95d:55017

5. T. T. Tri, The irreducible modular representations of semigroups of all matrices, Acta Math. Vietnamica 20 (1995), 43-53. MR 96f:20103

6. T. T. Tri, On a conjecture of Grant Walker for the first occurrence of irreducible modular representations of general linear groups, submitted.

7. R. M. W. Wood, Problems in the Steenrod algebra, Bull. London Math. Soc. 30 (1998), 449-517. CMP 99:01

Department of Mathematics, College of Sciences, University of Hue, Dai hoc Khoa hoc, Hue, Vietnam

E-mail address: paminh@bdvn.vnd.net 